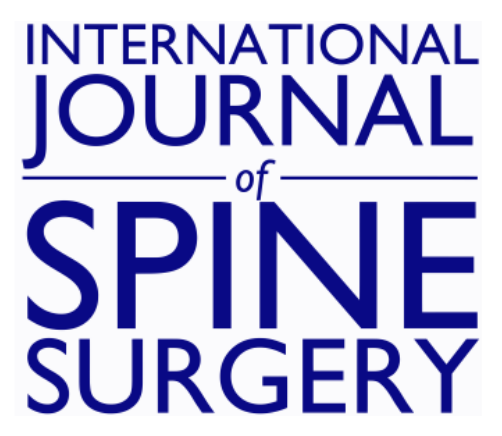

\title{
Hemangioma of the Lumbar Spine Involving the Spinous Process: A Rare Case Report and Review of the Literature
}

Faisal Mohammed Saleh Konbaz, Sami Ibrahim Al Eissa, Suhail Saad Alassiri, Anouar Bourghli, Majed Salah Abaalkhail, Muhammad Ejaz Ahmed, Fahad Helal Al Helal and Abdullah Omar Al Mahayni

Int J Spine Surg 2021, 14 (s4) S57-S65

doi: https://doi.org/10.14444/7166

http://ijssurgery.com/content/14/s4/S57

This information is current as of April 26, 2023.

Email Alerts Receive free email-alerts when new articles cite this article. Sign up at: http://ijssurgery.com/alerts 


\title{
Hemangioma of the Lumbar Spine Involving the Spinous Process: A Rare Case Report and Review of the Literature
}

\author{
FAISAL MOHAMMED SALEH KONBAZ, MBBS, ${ }^{1}$ SAMI IBRAHIM AL EISSA, MBBS, ${ }^{1}$ SUHAIL SAAD \\ ALASSIRI, MBBS, ${ }^{1}$ ANOUAR BOURGHLI, MBBS, ${ }^{2}$ MAJED SALAH ABAALKHAIL, MBBS, ${ }^{1}$ MUHAMMAD \\ EJAZ AHMED, MBBS, ${ }^{1}$ FAHAD HELAL AL HELAL, MBBS, ${ }^{1}$ ABDULLAH OMAR AL MAHAYNI, MBBS ${ }^{1}$ \\ ${ }^{I}$ National Guard Hospital, King Abdulaziz Medical City, Riyadh, Saudi Arabia, ${ }^{2}$ Kingdom Hospital, Riyadh, Saudi Arabia
}

\begin{abstract}
Hemangiomas of the spine are usually benign and asymptomatic. They can cause devastating complications such as pathological fractures of the spine and neurological disability. This report documents an atypical location of a hemangioma in a lumbar spinous process, in combination with a spondylolisthesis at the same level, which makes it even more uncommon. Surgery can be effective and safe and can significantly improve patient outcomes. Moreover, prior embolization can prevent acute hemorrhage in addition to providing careful diagnosis and evaluation.
\end{abstract}

Tumor

Keywords: hemangioma, vertebral body hemangioma, spinous process hemangioma, embolization, surgery, spondylolisthesis

\section{INTRODUCTION}

Hemangiomas are the most common benign neoplasms of the spine and are observed in approximately $11 \%$ of autopsies. ${ }^{1}$ They are usually asymptomatic and originate in the vertebral body with a reported incidence of $26 \% .^{2}$ Aggressive vertebral hemangiomas can extend from the vertebral body to the posterior vertebral arch but rarely originate from the spinous process without vertebral body involvement. ${ }^{3}$ Only a few spinous process hemangioma cases have been documented in the literature. ${ }^{4-9}$ This report presents a rare case of a 60 year-old woman complaining of lower back pain and inability to walk in relation to an aggressive spinous process hemangioma.

\section{MATERIALS AND METHODS}

A 60-year-old woman with diabetes mellitus, dyslipidemia, and asthma initially presented with pain localized in the lower back region. She did not seek medical care at the time of the onset of her symptoms. Three months after the pain started, the patient gradually became wheelchair bound due to the pain. In fact, the pain progressively began to radiate to both of her lower limbs, accompanied by paresthesia (specifically a burning sensation). The pain was exacerbated by movement (more with back extension) and was temporarily relieved by analgesics. The pain also woke her up at night. The patient did not complain of bowel/urinary incontinence or saddle anesthesia. She denied having any constitutional symptoms. Besides fatigue, she did not report any history of trauma. Because the pain persisted and gradually progressed, she presented to the emergency department at our hospital. Given her status, she was admitted for investigations and management and provided informed consent. On examination, the patient looked well and was afebrile, and vital signs were stable. She had no skin changes, obvious deformities, abnormal hair distribution, or temperature change in her back. On palpation of her lower back, there was no tenderness. Power and sensation in her right lower limb was normal. Power in the left lower limb had decreased to grade 4 in the iliopsoas and quadriceps muscles, whereas sensation was intact throughout. Upper limb examination was unremarkable. There were no signs of upper motor neuron lesions. Her gait could not be assessed because she was unable to walk due to the pain. Full oncological and infectious assessments were performed including tumor markers, which were negative. The patient's erythrocyte sedimentation rate was $54 \mathrm{~mm} / \mathrm{h}$ and her C-reactive protein level was $10 \mathrm{mg} / \mathrm{L}$. Lumbar spine x-ray revealed loss of normal outline of the spinous 
(A)

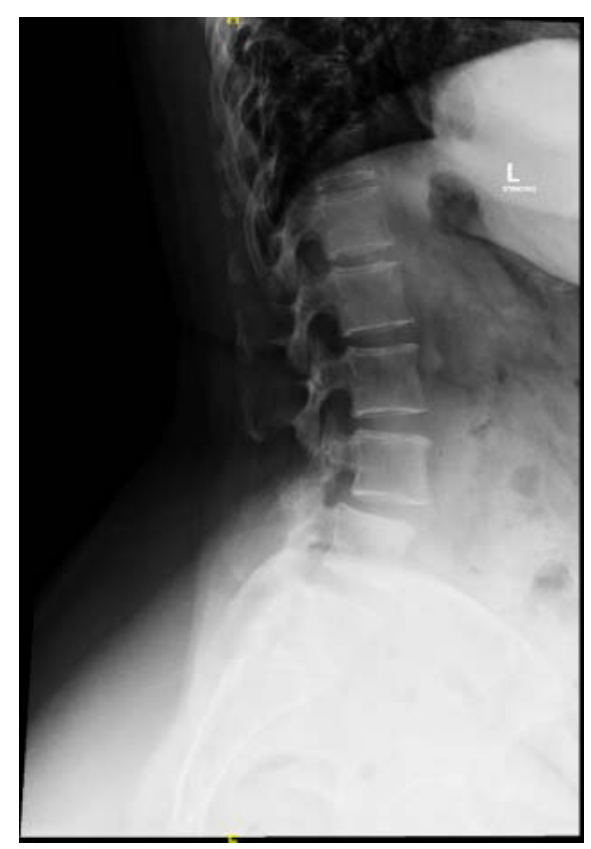

(B)

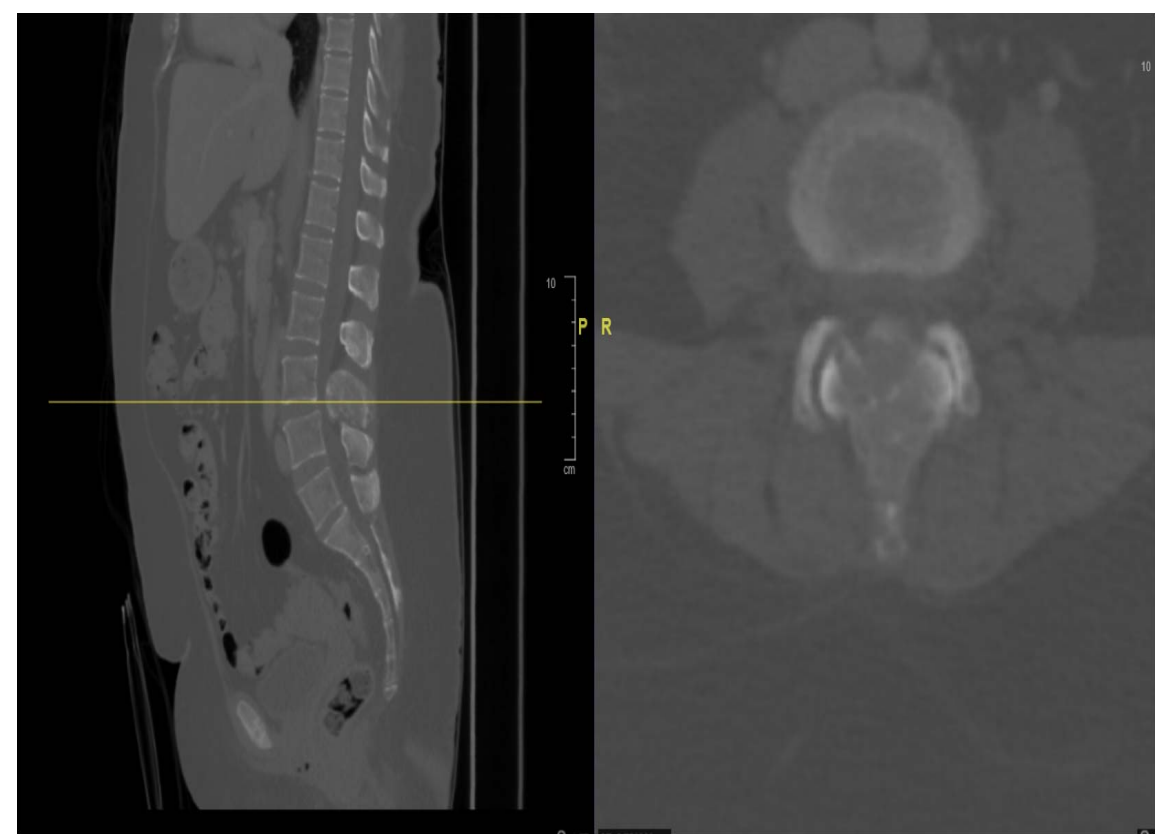

(C)

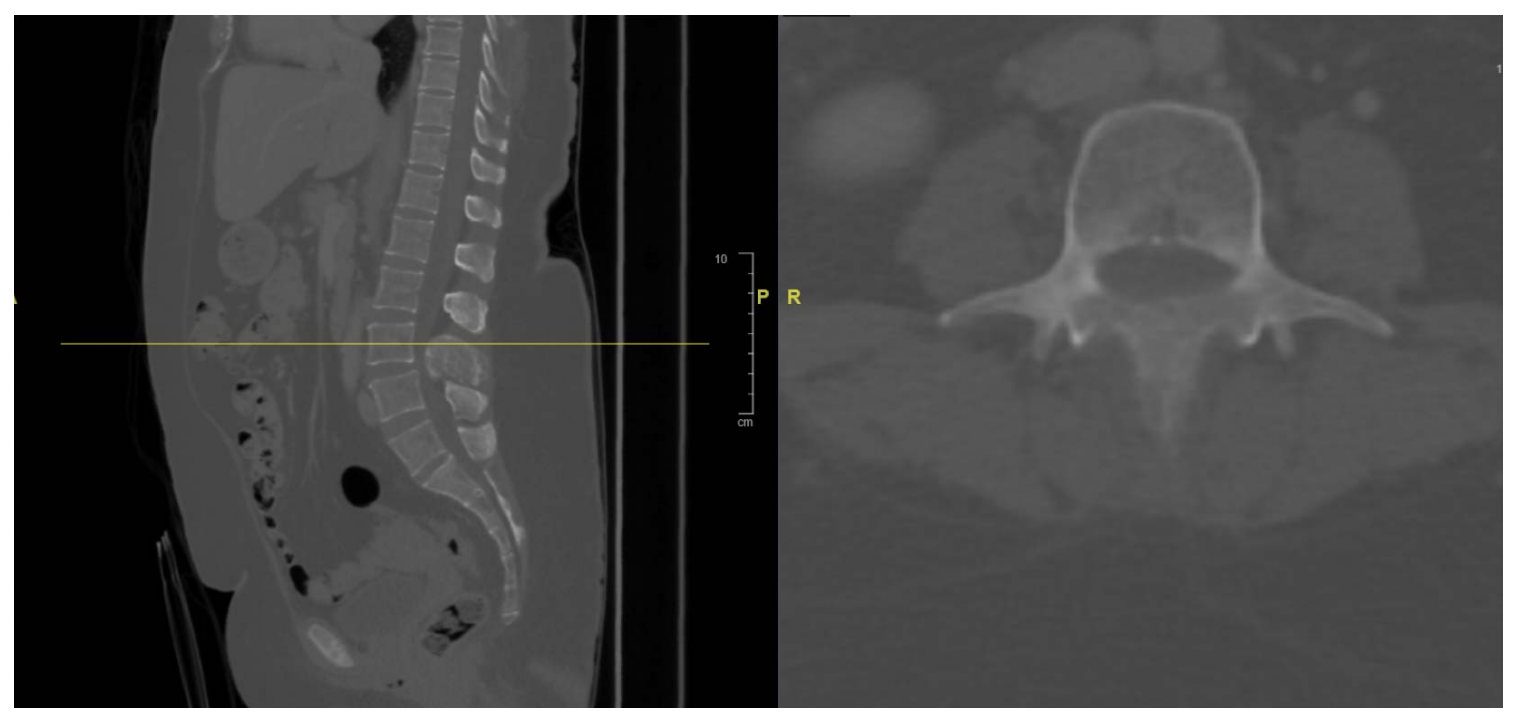

Figure 1. (A) Sagittal lumbar spine x-ray demonstrates loss of normal outline of the spinous process. (B, C) Sagittal and axial spine computed tomography (CT), respectively, demonstrating a lesion involving the lamina and spinous process of L3 vertebra.

process of L3 with L3-L4 spondylolisthesis grade 1 and sacralization of L5 (Figure 1A). Chest, abdomen, and pelvis computed tomography (CT) scans were done and revealed an expansile mixed sclerotic/lytic lesion involving the lamina and spinous process of the L3 vertebra (Figure 1B and 1C).

A bone scan showed focal avid uptake at L3. A lumbar spine MRI revealed an expansile contrastenhancing lesion involving the L3 spinous process with lamina involvement on both sides (Figure 2).
The lesion extended to the posterior epidural space and compressed the thecal sac, resulting in severe narrowing of the central canal at this level. The lesion inside the epidural space measured $3.3 \times 3.7 \times$ $2.6 \mathrm{~cm}$. Another lesion that looked like a benign hemangioma was also noted within the T12 vertebral body. A CT-guided biopsy from the L3 spinous process was performed, and results confirmed an intraosseous hemangioma. Given the clinical presentation with neurological deficit and 
(A)

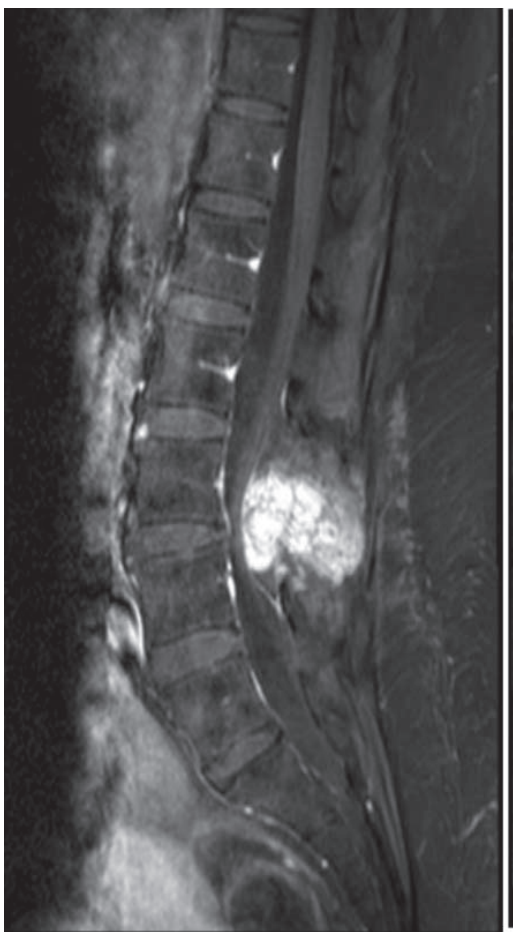

(B)

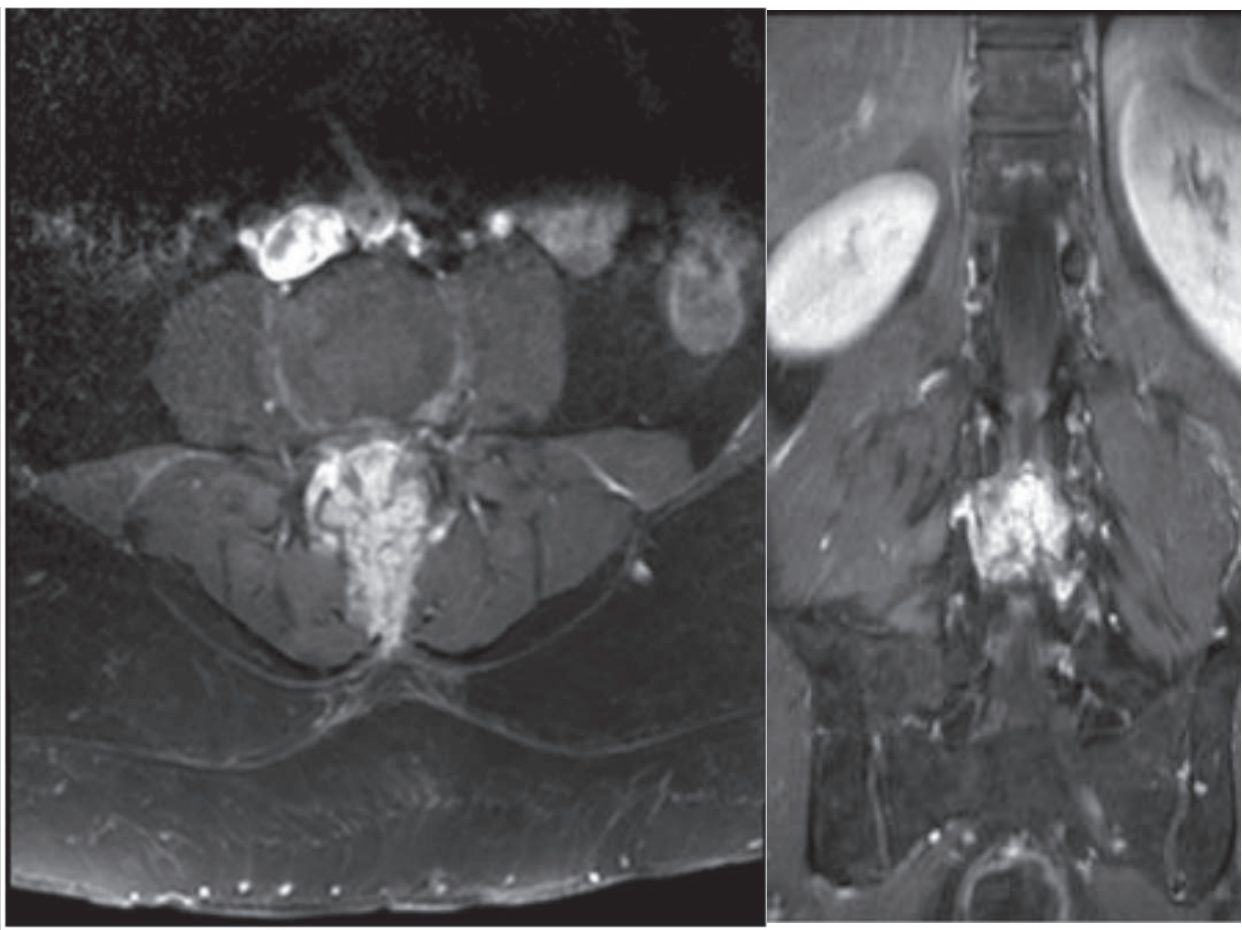

Figure 2. (A) T1-weighted sagittal lumbar spine MRI with contrast showing expansile enhancing lesion involving the spinous process of L3. (B) T1-weighted postcontrast axial image of the L3 lumbar vertebra. (C) T1-weighted coronal MRI with contrast.

the confirmation of the L3 posterior arch aggressive hemangioma with epidural extension, associated with L3-L4 spondylolisthesis, surgical management was decided consisting of L2 to L4 posterolateral fusion with decompressive laminectomy by wide resection of L3 posterior elements including the spinous process. Before surgery, and to avoid any major bleeding from the tumor during the procedure, spinal angiogram and embolization were performed through the right common femoral artery. Bilateral segmental arteries angiograms were performed at the L2 and L3 levels and the common iliac arteries. Tumor blush and aneurysmal dilatation of the distal radicular artery were demonstrated upon L3 segmental artery injection. Embolization was performed using onyx and polyvinyl alcohol particles, achieving complete obliteration of the aneurysm and a significant decrease in vascularity. There was no significant residual abnormal vascularity following the procedure (Figure 3). The patient was taken to the operating room immediately after embolization. The level was confirmed
(A)

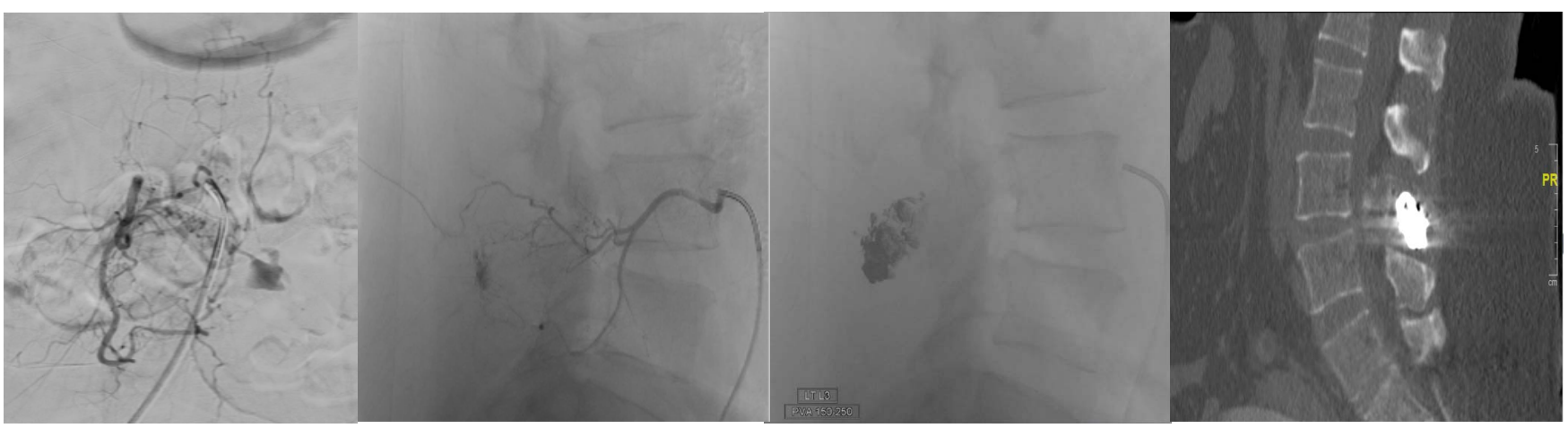

Figure 3. (A) Right L3 segmental artery angiogram anteroposterior view demonstrating abnormal vascular blush and contrast pooling consistent with hemangioma (B) Lateral view angiogram. (C) Fluoroscopic spot image obtained during spinal angiogram demonstrated embolization material (Onyx 18) in the spinous process lesion. (D) Spine computed tomography (CT) sagittal view showing embolization material (Onyx 18) seen in the spinous process of the L3 vertebra (postembolization). 


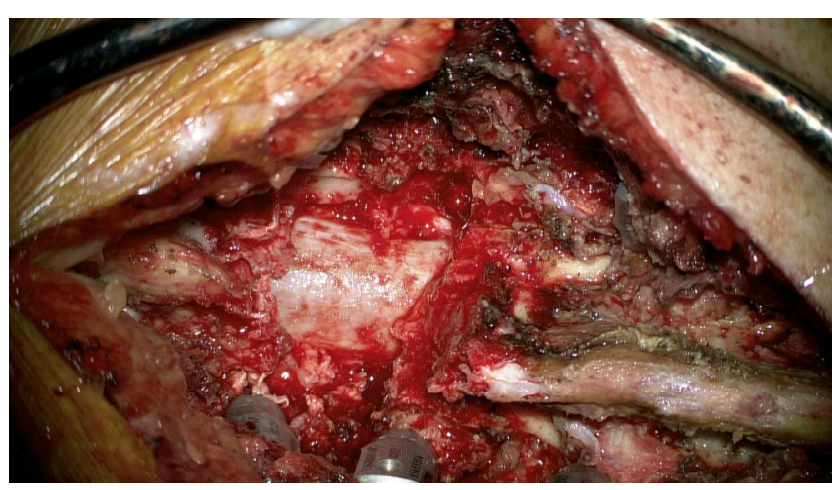

Figure 4. Intraoperative image after decompression and excision of the tumor.

under intraoperative x-ray guidance, and decompressive laminectomy was performed using a microscope (Figure 4). Decompression of the canal was achieved with complete excision of the tumor in a piecemeal technique. Once the tumor was completely excised involving the spinous process; bilateral lamina, bilateral superior, and inferior articular process at L3-L4; and the medial wall of the left L3 pedicle, we examined the dura and the L3 and L4 nerve roots to ensure proper decompression. Pedicle screws were inserted before decompression bilaterally from L2 to L4 but skipping the left L3 pedicle because the tumor was mainly in the spinous process extending to the lamina and the left L3 pedicle. Estimated blood loss intraoperatively was around $350 \mathrm{~mL}$. The postoperative pathologic diagnosis was conventional intraosseous hemangioma (Figure 5). Postoperative lumbar spine CT showed spinal fixation extending from L2 to L4 with 3 right pedicular screws and 2 left pedicular screws (Figure 6). Postoperative lumbar spine MRI indicated L2, L3, and L4 spinal fixation in good position with adequate decompression. Postoperative lumbar spine $x$-ray is shown in Figure 7.

Postoperative neurological examination of the patient demonstrated immediate improvement in her left lower limb function with a motor power of grade 5 .

The patient's lower back pain improved significantly within a few days following surgery. She could walk with the help of a walking frame and was discharged home on postoperative day 5. She completed her regular appointments and course of physiotherapy and was discharged from physiotherapy with instructions for home exercises involving strengthening and stretching exercises. She was seen 3 months postoperatively walking without assis-

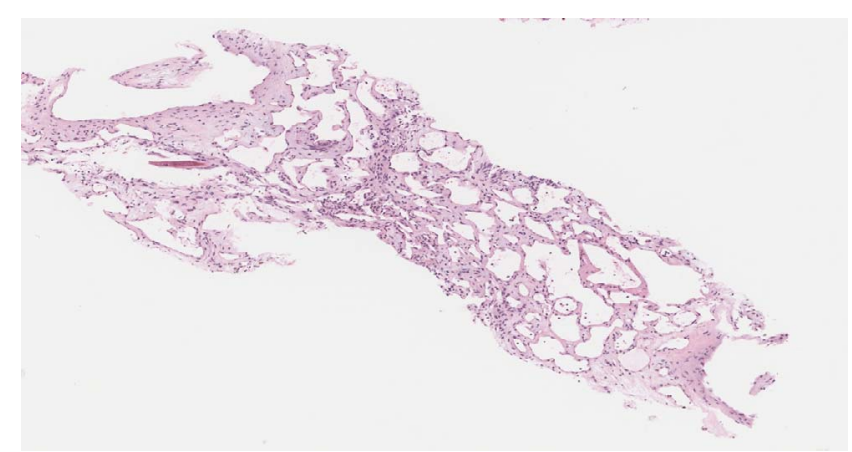

Figure 5. Histological examination reveals variable sizes of cystically dilated, thin-walled blood vessels that are lined by bland endothelial cells. No marked atypia, mitosis, or necrosis seen. Diagnosis: bland vascular proliferation consistent with hemangioma.

tance and presented satisfactory clinical and radiological results at 2 years (Figure 8).

\section{DISCUSSION}

Primary tumors of the vertebral column are relatively rare compared with secondary tumors, amounting to only approximately $10 \%$ of all vertebral column tumors. The most common benign tumors of the spine in adults are hemangiomas, which account for $20 \%-30 \%$ of benign vertebral column tumors. ${ }^{3,4}$ The majority of hemangiomas in the vertebral column are asymptomatic, and only $5 \%$ cause symptoms. Patients who are symptomatic complain mostly of nocturnal back pain. Most patients with vertebral hemangiomas are in their fourth to sixth decades of life, and women are affected twice as often as men. The hemangioma usually originates from the lower thoracic or lumbar region, and up to $30 \%$ of patients have multiple lesions. ${ }^{3,4}$ MRI usually demonstrates low-intensity $\mathrm{T} 1$ signals and high-intensity $\mathrm{T} 2$ signals, which is be explained by the low fat and high vascular components of the lesions, but ruling out more serious pathologies, particularly metastatic lesions or malignancies, is recommended. Hemangioma almost always originates from the vertebral body and may rarely extend into or originate from the posterior column of the vertebra. Hemangioma originating from the spinous process is extremely rare. In fact, during the past 2 decades, only 6 cases of spinous process hemangiomas have been reported in the literature. Patients were from 31 to 68 years old (5 women and 1 man). The spinous process hemangioma was located in the thoracic spine in 5 patients, and in the lumbar spine in 1 patients. Symptoms revealed either isolated back pain at the level of the lesion, or pain in combination with 
(A)

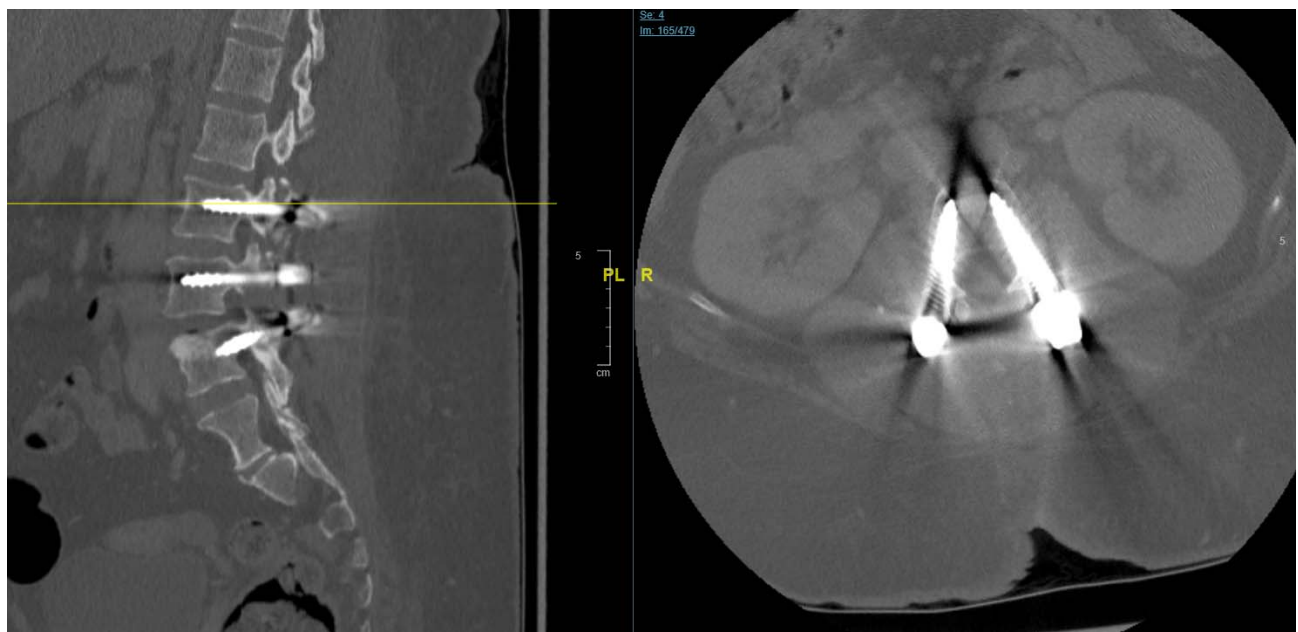

(B)

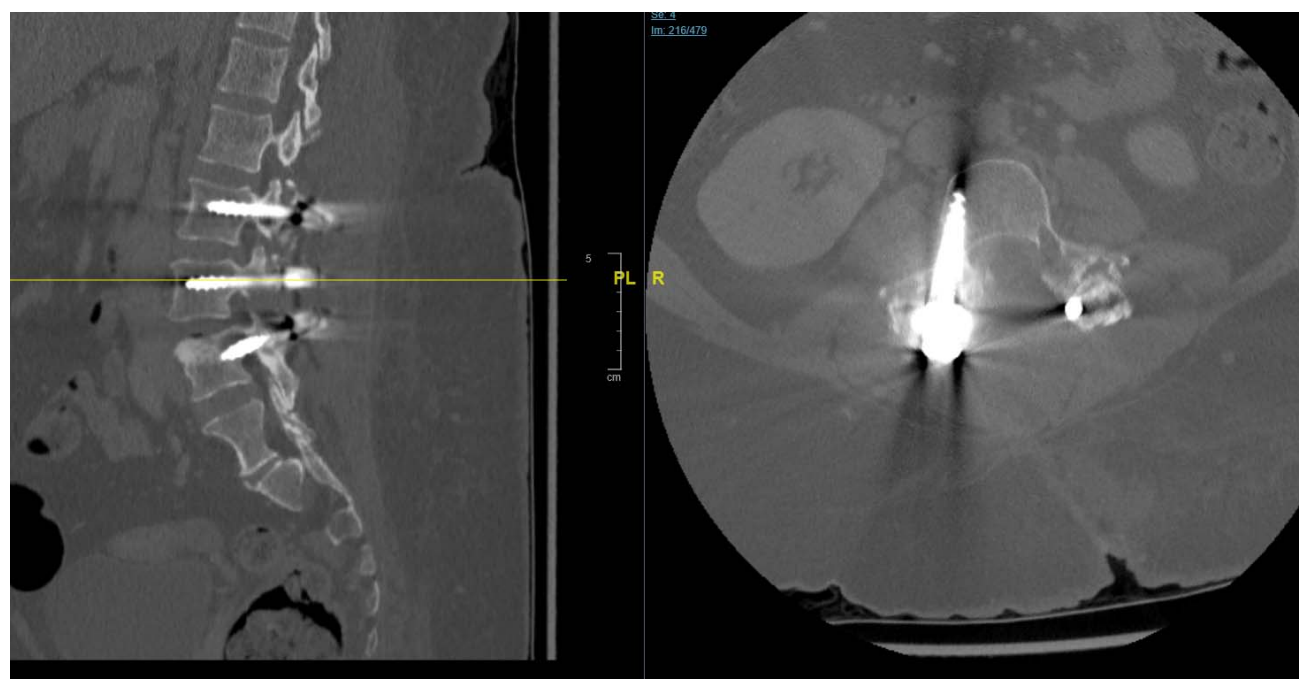

Figure 6. (A) Postoperative sagittal and axial computed tomography (CT) spine at L2 pedicle. (B) Postoperative sagittal and axial CT spine at L3 pedicle. (C-E) Postoperative sagittal and axial CT spine at L4 pedicle. Axial image showing the amount of tumor that was resected including the medial wall of the left L3 pedicle.

lower limb neurological deficit (paraparesis). All patients underwent surgical management consisting of either resection of the posterior elements alone or in combination with spinal fusion. ${ }^{4-9}$

Preoperative embolization has been widely described in the literature for the management of vertebral hemangioma to avoid major intraoperative bleeding and decrease the global amount of blood loss. This procedure was described in only 1 of the spinous process hemangioma cases, in which an open excisional biopsy was performed and blood loss was $900 \mathrm{~mL}$; due to the close proximity of a prominent radiculomedullary artery seen during angiography, embolization was aborted. ${ }^{11} \mathrm{We}$ nevertheless wanted in the case of our patient to avoid any major bleeding during surgery; this is why preoperative embolization was performed and was successful in reaching an acceptable intraoperative total blood loss of $350 \mathrm{~mL}$.

Postoperative radiotherapy can be an option in the treatment of vertebral hemangioma. ${ }^{12,13}$ It can be used as a single treatment in case of painful hemangioma without neurological symptoms and when surgical resection or interventional radiology procedures may result in high morbidity. ${ }^{12}$ It can also be used as a complementary treatment after tumor resection as illustrated in 2 of the spinous process hemangiomas cited in the literature; nevertheless, these cases were described 2 decades ago, whereas all other cases later on did not use radiotherapy in the case of complete tumor resection, and the current literature recommends radiotherapy mainly as a sole treatment when surgery is not indicated, when the patient is at risk of high 
(C)

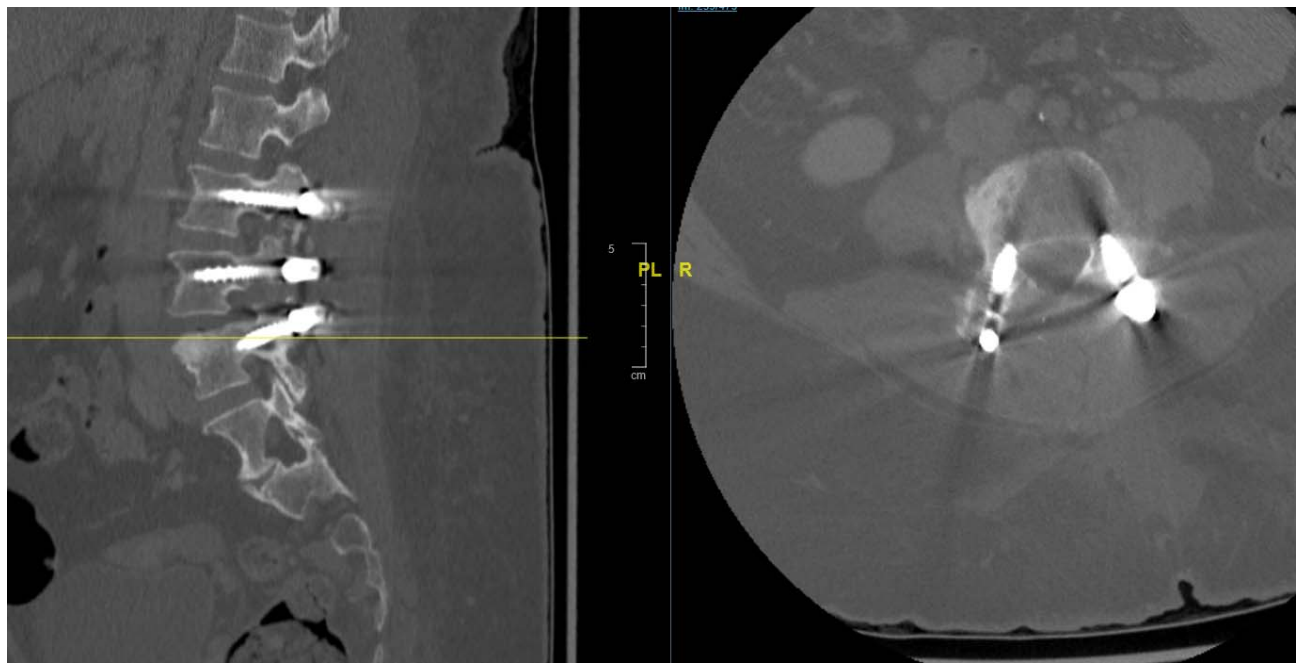

(D)

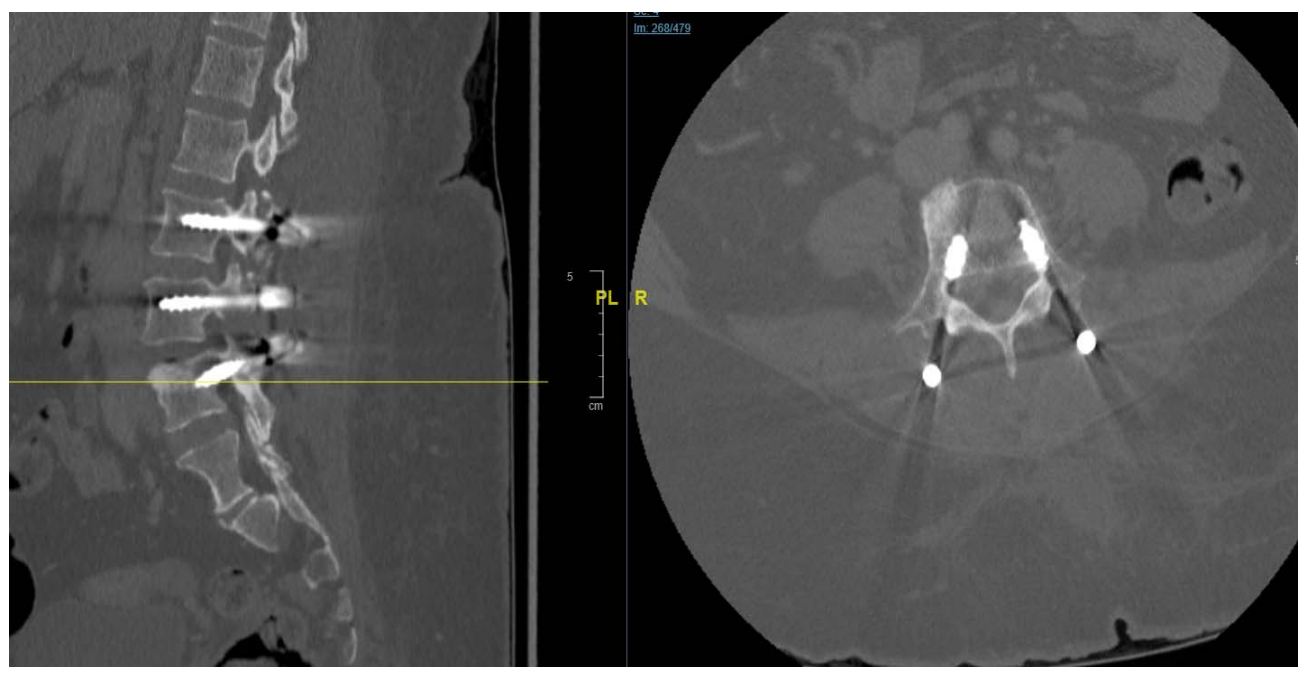

(E)

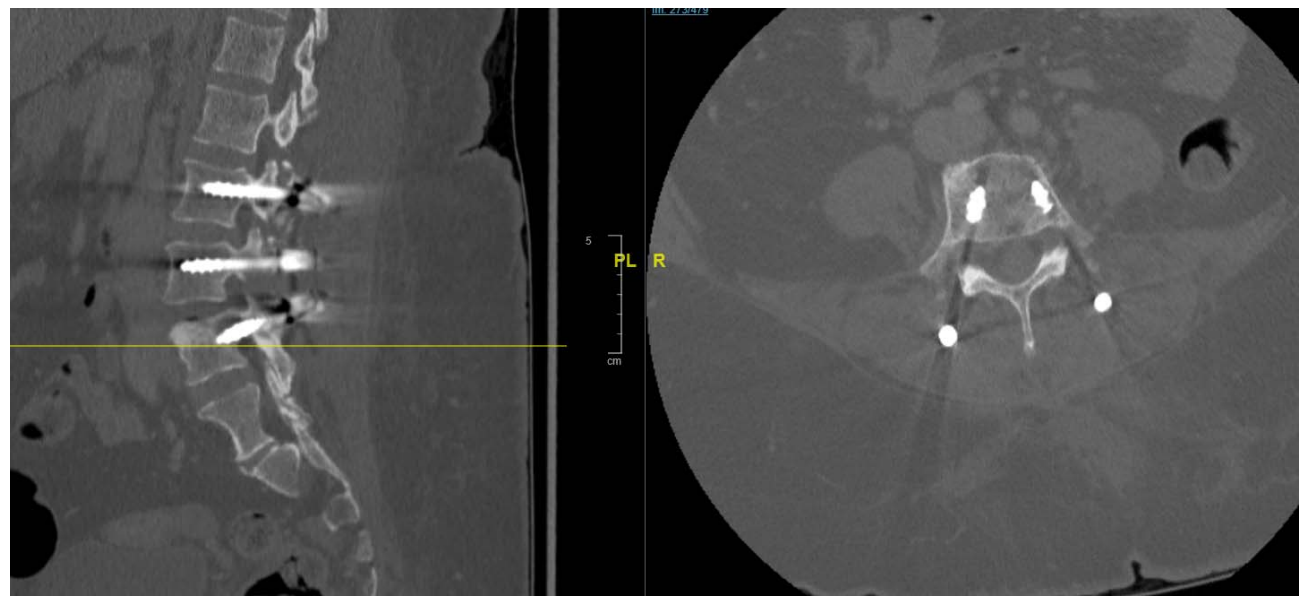

Figure 6. Continued. 
(A)

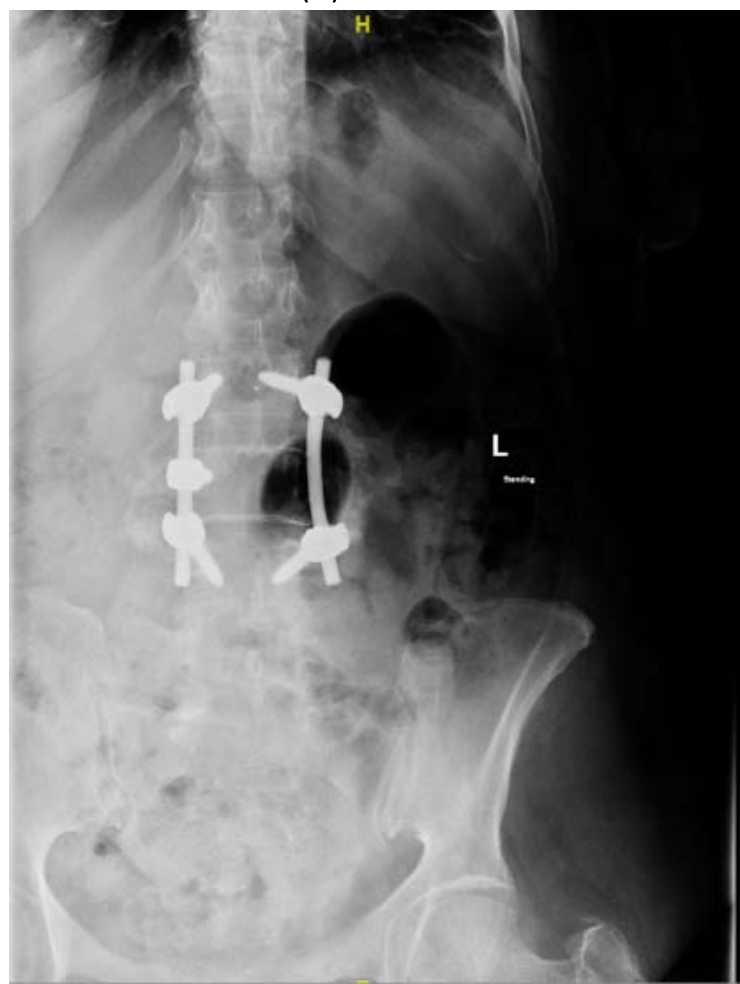

(B)

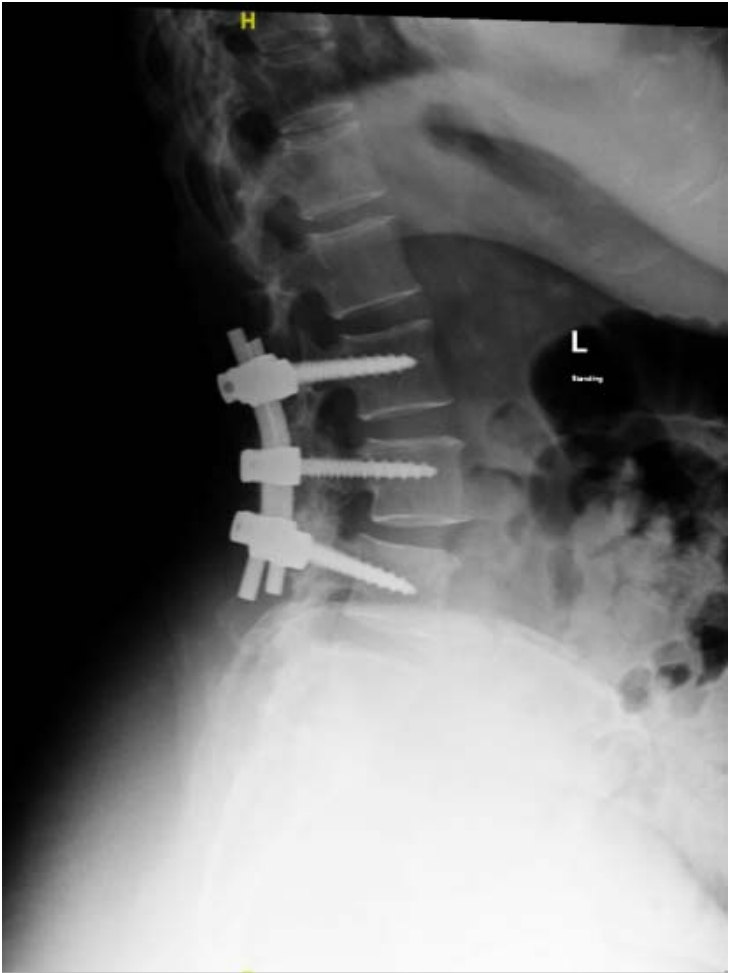

Figure 7. (A, B) Anteroposterior and lateral postoperative x-rays, respectively, showing 2 rods and transpedicular screws seen transfixing the vertebral body from L2 to L4. No evidence of looseness or alignment deformity. There is no pedicle screw on the left L3 vertebra.

(A)

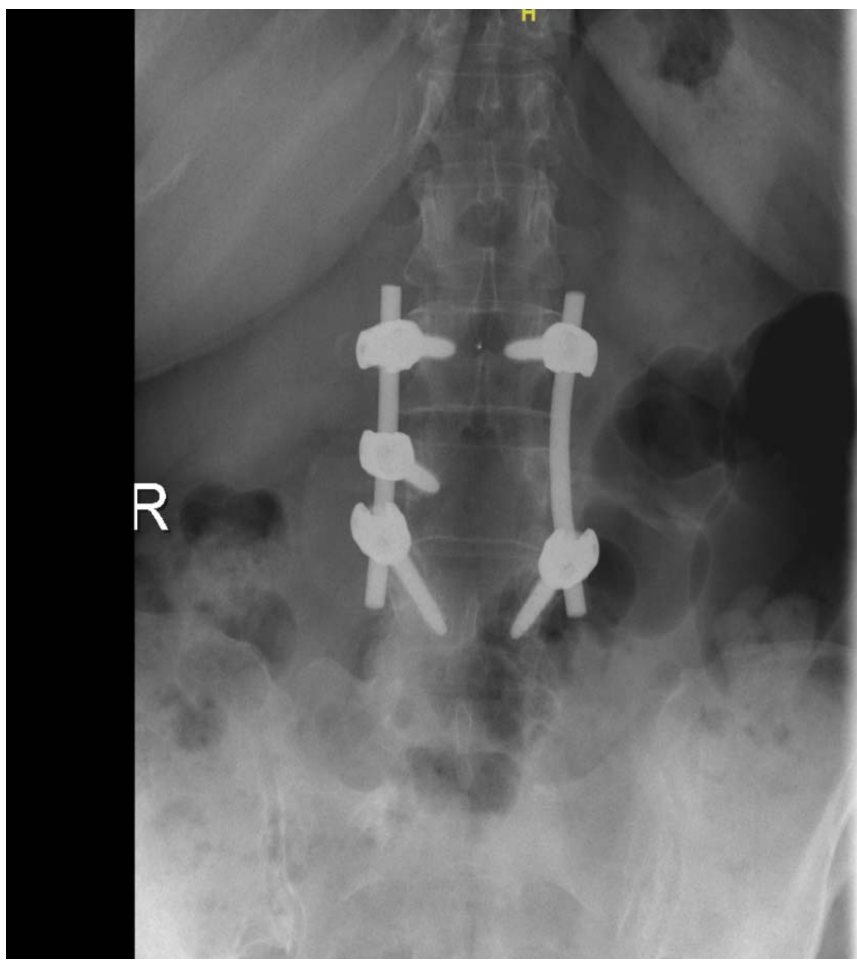

(B)

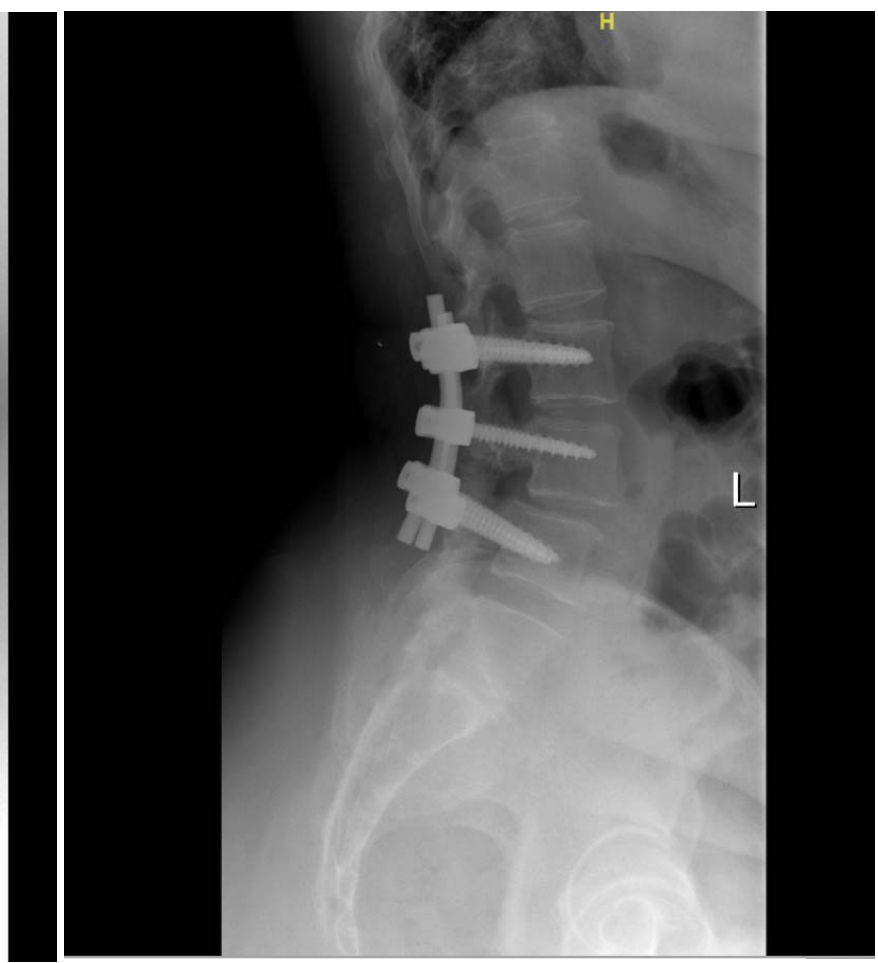

Figure 8. (A, B) Anteroposterior and lateral views of follow-up x-rays, respectively, 2 years postoperatively showing 22 rods and transpedicular screws seen transfixing the vertebral body from L2 to L4. No evidence of looseness or alignment deformity. There is no pedicle screw on the left $\mathrm{L} 3$ vertebra. 


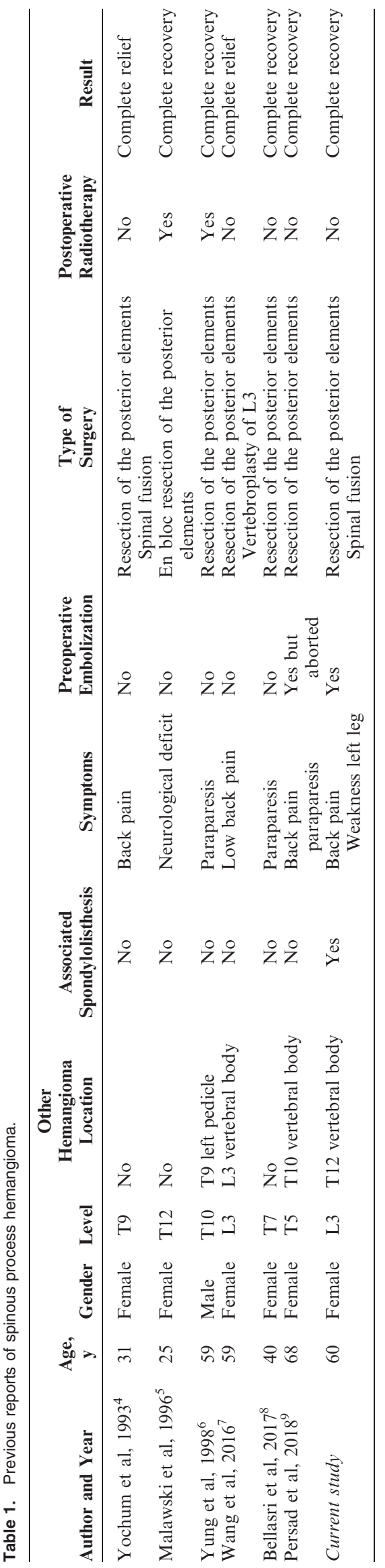

morbidity, or when surgical resection is incomplete. This is why in the case of our patient, no postoperative radiotherapy was performed, and the patient remained free of any recurrence at 2 years follow-up.

The originality of our case resides in the presence of a spinal instability at the level of the hemangioma, which had never before been described in the literature. In fact, our patient showed L3 spinous process hemangioma in association with L3-L4 degenerative spondylolisthesis. The presence of such an entity in addition to the tumor needed to be taken into account during the surgical management of the hemangioma. Therefore, spinal fixation needed to be performed in addition to the usual decompression by posterior elements resection. This is why in our case, posterior fixation with pedicle screws 1 level above and 1 level below the hemangioma was done.

All the patients in their last follow-up showed a complete relief from pain with full recovery of their initial neurological symptoms. The aforementioned reports are summarized in Table 1 . The prognosis of vertebral hemangiomas in general is favorable, but the prognosis of spinous process hemangiomas is not well known because only case reports have been described so far. Nevertheless, all mentioned patients demonstrated full recovery after treatment with no recurrence of the pathology at final followup.

\section{CONCLUSION}

Spinous process hemangiomas are very rare pathologies, and their presence with a spondylolisthesis at the same level is even rarer. They can become symptomatic, mainly causing pain, weakness, and paresthesia. After establishing the diagnosis via MRI, complete resection of the lesion with prior embolization if possible is advised, and additional spinal fixation with pedicle screws is recommended if a spondylolisthesis is associated.

\section{REFERENCES}

1. Doppman JL, Oldfield EH, Heiss JD. Symptomatic vertebral hemangiomas: treatment by means of direct intralesional injection of ethanol. Radiology. doi:10.1148/radiology. 214.2.r00fe46341

2. Peckham ME, Hutchins TA. Imaging of vascular disorders of the spine. Radiol Clin. 2019;57(2):307-318. doi:10. 1016/j.rcl.2018.09.005

3. Theodorou DJ, Theodorou SJ, Sartoris DJ. An imaging 
overview of primary tumors of the spine: part 1. Benign tumors. Clin Imaging. doi:10.1016/j.clinimag.2007.09.011

4. Yochum TR, Lile RL, Schultz GD, Mick TJ, Brown CW. Acquired spinal stenosis secondary to an expanding thoracic vertebral hemangioma. Spine (Phila Pa 1976). doi:10.1097/ 00007632-199302000-00021

5. Malawski S, Sokólski B. A case of spinal hemangioma uncommonly located within spinous processes and laminae of Th12. Chir Narzadow Ruchu Ortop Pol. 1996;61(1):47-51.

6. Yung BCK, Loke TKL, Yuen NWF, Chan CC. Spinal cord compression caused by thoracic vertebral hemangioma involving only the posterior elements of two contiguous vertebrae. Skeletal Radiol. 27, 169-172 (1998). doi:10.1007/ s002560050359

7. Wang L, Song Y. A rare case of symptomatic hemangioma of the lumbar spine involving the spinous process. Spine J. 2016;16(3):e191-2. doi:10.1016/j.spinee.2015.10.020

8. Bellasri S, Fatihi J, Elktaibi A, Asri AC El. Acute spinal cord compression caused by atypical vertebral hemangioma. $J$ Craniovertebr Junction Spine. doi:10.4103/jcvjs.JCVJS_14_17

9. Persad AR, Fox RJ, Rempel JL. Symptomatic vertebral hemangioma of the posterior elements sharing blood supply with a radiculomedullary artery. Int J Spine Surg. doi:10.14444/ 5049

10. Chi JH, Bydon A, Hsieh P, Witham T, Wolinsky JP, Gokaslan ZL. Epidemiology and demographics for primary vertebral tumors. Neurosurg Clin N Am. doi:10.1016/j.nec.2007. 10.005

11. Acosta FL, Dowd CF, Chin C, Tihan T, Ames CP, Weinstein PR. Current treatment strategies and outcomes in the management of symptomatic vertebral hemangiomas. Neurosurgery. doi:10.1227/01.NEU.0000194846.55984.C8

12. Parekh AD, Amdur RJ, Mendenhall WM, Morris CG, Zlotecki RA. Long-term tumor control with radiotherapy for symptomatic hemangioma of a vertebral body. Spine (Phila Pa 1976). doi:10.1097/BRS.0000000000002973

13. Wang B, Meng N, Zhuang H, Han S, Yang S, Jiang L, et al. The role of radiotherapy and surgery in the management of aggressive vertebral hemangioma: a retrospective study of 20 patients. Med Sci Monit. doi:10.12659/MSM.910439.

Disclosures and COI: The authors received no funding for this study and report no conflicts of interest.

Corresponding Author: Abdullah Omar Al Mahayni, Orthopedics Surgery Resident, National Guard Hospital, King Abdulaziz Medical City, PO Box 22490, Riyadh 11426, Saudi Arabia. Phone: 00966501737202; Email: abdullahmahayni@ hotmail.com.

Published 11 February 2021

This manuscript is generously published free of charge by ISASS, the International Society for the Advancement of Spine Surgery. Copyright (c) 2020 ISASS. To see more or order reprints or permissions, see http://ijssurgery.com. 